\title{
PRINCIPAIS ASPECTOS QUE FAVORECEM O DESENVOLVIMENTO DE INCONTINÊNCIA URINÁRIA EM PUÉRPERAS
}

\section{MAIN ASPECTS THAT FAVOR THE DEVELOPMENT OF URINARY INCONTINENCE IN WOMEN AFTER CHILDBIRTH}

\section{PRINCIPALES ASPECTOS QUE FAVORECEN EL DESARROLLO DE INCONTINENCIA URINARIA EN MUJERES DESPUÉS DEL PARTO}

\begin{abstract}
Miguir Terezinha Vieccelli Donoso할 Elisângela Soares da Silva Reis ${ }^{2}$; Torcata Amorim³; Salete Maria de Fátima Silqueira ${ }^{4}$; Elis Regina Silva Pinheiro ${ }^{5}$; Selme Silqueira de Matos ${ }^{6}$

\section{RESUMO}

Objetivo: Identificar principais fatores que favorecem o desenvolvimento de incontinência urinária em mulheres no período pós parto. Método: O referencial metodológico adotado foi a Revisão Integrativa de Literatura. A estratégia de busca foi realizada nas bases Medline, IBECS e Lilacs, em abril de 2017. Os critérios de inclusão foram: artigos resultantes de pesquisa primária, quantitativos, nos idiomas português, inglês ou espanhol, publicados entre o período de 2012 a 2017 e que contemplassem aspectos relacionados à incontinência urinária no puerpério. Foram selecionados 18 artigos, por atenderem os critérios de inclusão desta pesquisa. Resultados: Os fatores que mais favorecem a incontinência urinaria no pós-parto são: alterações fisiológicas da gravidez, condições preexistentes e falta de orientações à gestante, além do não preparo da musculatura pélvica durante a gestação. Conclusão: Os estudos reiteram a importância de um prénatal bem assistido, com equipe multidisciplinar, incluindo o enfermeiro estomaterapeuta, uma vez que a estomaterapia abrange as áreas de estomas, feridas e incontinências. Estes profissionais devem despertar na gestante o interesse pela qualidade de vida no pós parto.

Descritores: Incontinência urinária; Período Pós-parto; Gravidez.
\end{abstract}

${ }^{1}$ Doutora em Ciências da Saúde pela Faculdade de Medicina da UFMG. Professor associado do Departamento de Enfermagem Básica da Escola de Enfermagem da UFMG (autor correspondente). Fone (31) 34099177. E.mail: miguir@enf.ufmg.br

${ }^{2}$ Estomaterapeuta pela Escola de Enfermagem da Universidade Federal de Minas Gerais (UFMG). Enfermeira do Hospital Sofia Feldman da cidade de Belo Horizonte, MG.

${ }^{3}$ Doutora em Enfermagem pela Escola de Enfermagem da Universidade de São Paulo (USP). Professor adjunto do Departamento de Enfermagem Materno Infantil e Saúde Pública da Escola de Enfermagem da UFMG.

${ }^{4}$ Doutora em Saúde pública pela Escola de Enfermagem da USP (Ribeirão Preto, SP). Professor associado do Departamento de Enfermagem Básica da Escola de Enfermagem da UFMG.

${ }^{5}$ Enfermeira obstétrica pela Escola de Enfermagem da UFMG. Enfermeira do Hospital Sofia Feldman da cidade de Belo Horizonte, MG. Responsável técnico pela área de Saúde da Mulher na Prefeitura Municipal de Betim, MG.

6 Doutora em Enfermagem pela Escola de Enfermagem da UFMG. Professor associado do Departamento de Enfermagem Básica da Escola de Enfermagem da Universidade Federal de Minas Gerais. 


\begin{abstract}
Objective: To identify the main factors that favor the development of urinary incontinence in postpartum women. Method: The adopted methodological framework was the Integrative Review of Literature. A search strategy was carried out in the Medline, IBECS, and Lilacs databases in April 2017. The inclusion criteria were: articles presenting results that contemplated aspects related to urinary incontinence during postpartum, based on primary research, quantitative, and published in Portuguese, English, or Spanish between 2012 and 2017. A total of 18 articles were selected. Results: The following factors were identified as the main factors that most favored postpartum urinary incontinence: physiological pregnancy alterations, preexisting conditions, lack of guidance, and failed pelvic muscles preparation during pregnancy. Conclusion: The studies reiterate the importance of well-tended prenatal care provided by a multidisciplinary team that includes a stomato-therapist nurse because stoma therapy encompasses the care of stomas, wounds, and incontinence. These professionals should awaken the interest for an improved quality of life in the postpartum period in pregnant women.

Descriptors: Urinary incontinence; Postpartum Period; Pregnancy.
\end{abstract}

\title{
RESUMEN
}

Objetivo: Identificar los factores principales que favorecen el desarrollo de incontinencia urinaria en mujeres en el período post-parto. Método: El referencial metodológico adoptado fue la Revisión Integrativa de Literatura. La estrategia de búsqueda se realizó en Medline, Lilacs y IBECS en abril de 2017. Los criterios de inclusión fueron: artículos resultantes de la investigación primaria, cuantitativa, escrito en portugués, Inglés o Español, publicados entre el período 2012-2017 y que contemplaran aspectos relacionados a la incontinencia urinaria en el en el período posparto. Se seleccionaron 18 artículos para cumplir con los criterios de inclusión de esta investigación. Resultados: Los factores identificados como los que más favorecen la incontinencia urinaria en el posparto fueron alteraciones fisiológicas del embarazo, condiciones preexistentes y falta de orientaciones a mujeres embarazadas, además de la no preparación de la musculatura pélvica durante la gestación. Conclusión: El estudio reitera la importancia de un prenatal bien asistido, con equipo multidisciplinario, incluyendo el enfermero estomaterapeuta. Estos profesionales deben despertar el interés de la mujer embarazada por la calidad de vida después del parto.

Descriptores: Incontinencia urinaria; Periodo posparto; Embarazo.

\section{INTRODUÇÃO}

O período pós parto ou puerpério constitui-se como momento peculiar, demandando dos profissionais de saúde um comprometimento na avaliação e no cuidado dispensado durante este período à mãe, criança e família. Os determinantes mórbidos, diretamente, relacionados ao puerpério podem ser evitados por meio de ações de saúde, direcionadas à mulher nesse período. ${ }^{1}$

O pós-parto é por si só um período singular na vida da mulher. Trata-se de 
momentos em que esta se encontra sujeita a agravos como, por exemplo, a depressão, a dor perineal e a incontinência urinária. ${ }^{2}$

A incontinência urinária (IU) é definida como uma condição na qual ocorre queixa de qualquer perda involuntária de urina, sendo um problema social e higiênico. ${ }^{3}$

O tipo de IU mais comum no pósparto é a de esforço, seguida pela IU mista e IU de urgência. Em geral, a perda urinária nesta fase é pouco frequente e em pequena quantidade. $^{4}$

A IU no puerpério pode ser considerada um sério problema que interfere no trabalho, na vida social e na vida sexual das mulheres. ${ }^{4}$ No entanto, no Brasil, há poucos trabalhos científicos dedicados à IU nos períodos gestacional e puerperal, o que dificulta a identificação da população afetada e a comparação dos resultados. ${ }^{5}$ Este foi considerado o problema desta pesquisa.

Assim, este estudo teve como objetivo identificar fatores que favorecem o desenvolvimento de incontinência urinária em puérperas. Justifica-se que esta identificação poderá contribuir com o desenvolvimento de intervenções para evitar ou mesmo diminuir os episódios deste agravo, e por conseguinte, melhorar a qualidade de vida da mulher no puerpério.

\section{MÉTODO}

O referencial metodológico adotado neste estudo foi a Revisão Integrativa de Literatura. Esse tipo de estudo possibilita conclusões subsidiadas em evidências científicas a respeito de um assunto, corroborando uma tomada de decisão na prática clínica. ${ }^{6}$

Para a elaboração desta revisão, foram percorridas as seguintes etapas: identificação do tema ou questionamento da revisão integrativa, amostragem ou busca na literatura, categorização dos resultados e apresentação da revisão integrativa. ${ }^{6}$

A pergunta que norteou esse trabalho foi: quais aspectos favorecem $o$ desenvolvimento de IU em puérperas? Para levantamento da literatura, realizou-se busca na Biblioteca Virtual em Saúde (BVS). Foram utilizados os descritores: "Incontinência urinária" e "Período pósparto", utilizando-se os booleanos: AND e 
OR. A estratégia de busca realizada para seleção dos artigos foi a seguinte:

Quadro 1: Estratégia de busca realizada na BVS. Abril 2017. (mh:("Incontinência Urinária")) AND (mh:("Período Pós-Parto")) AND (year_cluster:("2012" or "2013" or "2014" or "2015" or "2016" or "2017"))

Como critérios de inclusão, os estudos deveriam ter sido publicados na forma de artigo, resultantes de pesquisa primária, quantitativos, nos idiomas português, inglês ou espanhol, publicados entre o período de 2012 a 2017 e que contemplassem aspectos relacionados à IU no puerpério.

A busca dos artigos se deu durante o mês de abril de 2017. As bases de dados contempladas na BVS foram: Medical Literature Analysis and Retrieval System Online (Medline): 42 artigos; Indice Bibliográfico Espanhol de Ciências da Saúde (IBECS): 05 artigos e Literatura LatinoAmericana em Ciências da Saúde (LILACS): 05 artigos. Os resumos destes 52 artigos foram lidos, tendo sido excluídos 34 por apresentarem-se repetidos ou com abordagem que não contemplava a questão norteadora ou ainda por apresentarem método de pesquisa de revisão integrativa literária e pesquisas qualitativas. Dessa forma, 18 artigos constituíram essa revisão integrativa de literatura.

Como referencial teórico, utilizou-se a Prática Baseada em Evidências - PBE. Evidência significa a constatação de uma verdade que não gera qualquer dúvida, acrescentam que a evidência científica representa uma comprovação de que um determinado conhecimento é verdadeiro ou falso, ressalta-se ainda que para a validação desta se faça necessária uma pesquisa prévia, conduzida dentro dos preceitos científicos. ${ }^{7}$

Para se aferir o nível de evidência das pesquisas apresentadas recomenda-se realizar uma classificação de acordo com o delineamento metodológico apresentado. Essa classificação pode ser assim visualizada: $^{7}$ 
Nível I: Metanálise de estudos controlados randomizados.

Nível II: Estudo individual com delineamento experimental controlado randomizado.

Nível III: Estudo quase experimental como grupo único, não randomizados, controlado, com pré e pós-teste ou estudos emparelhados tipo caso controle.

Nível IV: Estudo não experimental: descritivos ou com abordagem metodológica qualitativa ou estudo de caso.

Nível V: Relatório de casos ou dados obtidos sistematicamente, de qualidade verificável ou dados de programas de avaliação.

Nível VI: Opinião de especialistas na área, de órgãos de regulamentação ou legais.

\section{RESULTADOS}

Os 18 artigos selecionados tiveram como autores médicos, enfermeiros e fisioterapeutas, sendo a maioria de autoria médica. Em relação ao ano de publicação, dois artigos foram publicados em 2015, três em 2014, sete em 2013 e seis em 2012.

Quanto aos níveis de evidência, quatro artigos apresentaram nível de evidência II e 14 artigos, nível de evidência IV.

A súmula dos artigos que compuseram esta revisão encontra-se a seguir, na forma de quadro sinóptico. Estes foram codificados em Artigo 1 a Artigo 18, para facilitar a localização dos mesmos. 
Quadro 2: Quadros sinópticos de Revisão Integrativa de Literatura. Abril 2017.

\begin{tabular}{|c|c|c|c|}
\hline Título da publicação & $\begin{array}{c}\text { Delineamento/Níve } \\
1 \text { evidência }\end{array}$ & Fatores que favorecem a IU no pós parto & Conclusões/Recomendações \\
\hline $\begin{array}{l}\text { Artigo } 01:{ }^{8} \text { Factors influencing } \\
\text { postpartum women's willingness to } \\
\text { participate in a preventive pelvic floor } \\
\text { muscle training program: a web-based } \\
\text { survey. }\end{array}$ & $\begin{array}{l}\text { Estudo transversal. } \\
\text { Nível de evidência: } \\
\text { IV }\end{array}$ & $\begin{array}{l}\text { 1) Falta de treinamento e falta de preparo da } \\
\text { musculatura pélvica na gestação. }\end{array}$ & $\begin{array}{l}\text { A importância dos Programas de treinamento para } \\
\text { fortalecimento do soalho pélvico é reconhecida } \\
\text { pelas mulheres, porém a adesão ao programa } \\
\text { depende do grau de comprometimento e } \\
\text { interferência na qualidade de vida ou à pratica de } \\
\text { hábitos de vida saudável. Além disso, outros fatores } \\
\text { como custo e tempo de deslocamento podem ser } \\
\text { considerados como desencorajadores para a adesão } \\
\text { ao programa de treinamento de força muscular do } \\
\text { soalho pélvico. }\end{array}$ \\
\hline $\begin{array}{l}\text { Artigo } 02:{ }^{9} \text { Consultation about urinary } \\
\text { and faecal incontinence in the year after } \\
\text { childbirth: a cohort study. }\end{array}$ & $\begin{array}{l}\text { Estudo de coorte } \\
\text { prospectivo. } \\
\text { Nível de evidência: } \\
\text { II }\end{array}$ & $\begin{array}{l}\text { 1) Falta de um acompanhamento de saúde adequado e } \\
\text { de orientações de cuidados primários nos primeiros } \\
12 \text { meses pós-parto. }\end{array}$ & $\begin{array}{l}\text { Há a necessidade de orientações quanto a } \\
\text { incontinência pós parto para que as mulheres } \\
\text { possam relatar seu problema e assim receber um } \\
\text { acompanhamento adequado. }\end{array}$ \\
\hline $\begin{array}{l}\text { Artigo } 03:{ }^{10} \text { Factors associated with } \\
\text { treatment-seeking behavior for } \\
\text { postpartum urinary incontinence. }\end{array}$ & $\begin{array}{l}\text { Estudo transversal. } \\
\text { Nível de } \\
\text { Evidencia: IV }\end{array}$ & $\begin{array}{l}\text { 1) Falta de aconselhamento sobre IU durante a } \\
\text { gravidez; } \\
\text { 2) Falta de exercício físico orientado no período pós- } \\
\text { parto; } \\
\text { 3) Paridade; } \\
\text { 4) Renda familiar. }\end{array}$ & $\begin{array}{l}\text { O aconselhamento e as orientações durante o pré- } \\
\text { natal podem reduzir a ocorrência de IU no pós parto. }\end{array}$ \\
\hline $\begin{array}{l}\text { Artigo } 04:{ }^{11} \text { Urinary incontinence during } \\
\text { pregnancy and postpartum. Associated } \\
\text { risk factors and influence of pelvic floor } \\
\text { exercises. }\end{array}$ & $\begin{array}{l}\text { Estudo descritivo, } \\
\text { observacional e } \\
\text { prospectivo. } \\
\text { Nível de } \\
\text { Evidência: IV }\end{array}$ & $\begin{array}{l}\text { 1) Ocorrência de IU ainda durante a gestação; } \\
\text { 2) Parto vaginal; } \\
\text { 3) Parto instrumental; } \\
\text { 4) Episiotomia; } \\
\text { 5) Idade; } \\
\text { 6) IMC. }\end{array}$ & $\begin{array}{l}\text { Exercícios físicos no soalho pélvico no período pós- } \\
\text { parto contribuem para a diminuição da IU pós parto. }\end{array}$ \\
\hline $\begin{array}{l}\text { Artigo } 05:^{12} \text { Prevalencia y factores de } \\
\text { riesgo de incontinencia urinaria en } \\
\text { mujeres que consultan por dolor } \\
\text { lumbopélvico: estudio multicéntrico. }\end{array}$ & $\begin{array}{l}\text { Estudo transversal. } \\
\text { Nível de evidência: } \\
\text { IV }\end{array}$ & $\begin{array}{l}\text { 1)Asma; } \\
\text { 2)Hipertensão; } \\
\text { 3)Constipação intestinal; } \\
\text { 4) Multiparidade. }\end{array}$ & Não apresenta recomendações. \\
\hline
\end{tabular}

Rev Enferm Health Care [Online]. Jan/Jul 2020; 9(1):144-159

ISSN 2317-1154 


\begin{tabular}{|c|c|c|c|}
\hline $\begin{array}{l}\text { Artigo } 06:{ }^{13} \text { Urinary incontinence and } \\
\text { weight changes during pregnancy and } \\
\text { post partum: a pending challenge. }\end{array}$ & $\begin{array}{l}\text { Estudo } \\
\text { observacional de } \\
\text { coorte. } \\
\text { Nível de } \\
\text { Evidência: II }\end{array}$ & $\begin{array}{l}\text { 1) IMC elevado; } \\
\text { 2) Manutenção de sobrepeso durante os seis primeiros } \\
\text { meses pós-parto. }\end{array}$ & $\begin{array}{l}\text { O aconselhamento individualizado sobre hábitos } \\
\text { alimentares e sobre prática de exercícios para evitar } \\
\text { sobrepeso após a gravidez podem ter um impacto } \\
\text { considerável na diminuição do risco IU. }\end{array}$ \\
\hline $\begin{array}{l}\text { Artigo } 07:{ }^{14} \text { Prevalence of urinary and } \\
\text { fecal incontinence in Chinese women } \\
\text { during and after their first pregnancy. }\end{array}$ & $\begin{array}{l}\text { Estudo prospectivo } \\
\text { observacional. } \\
\text { Nível de evidencia: } \\
\text { IV }\end{array}$ & $\begin{array}{l}\text { 1) Aumento do IMC no primeiro trimestre de } \\
\text { gravidez; } \\
\text { 2) Parto vaginal; } \\
\text { 3) Parto instrumental; } \\
\text { 4) Idade. }\end{array}$ & $\begin{array}{l}\text { Sendo a gravidez um fator de risco para o } \\
\text { desenvolvimento de incontinência urinária, } \\
\text { orientações quanto ao IMC, devem ser oferecidas a } \\
\text { mulher, pois este é um fator que contribui mas que } \\
\text { pode ser evitável. }\end{array}$ \\
\hline $\begin{array}{l}\text { Artigo } 08: 15 \text { Pelvic floor muscle training } \\
\text { program increases muscular contractility } \\
\text { during first pregnancy and postpartum: } \\
\text { electromyographic study. }\end{array}$ & $\begin{array}{l}\text { Estudo clínico, } \\
\text { prospectivo e cego } \\
\text { Nível de } \\
\text { Evidência: IV } \\
\end{array}$ & $\begin{array}{l}\text { 1) Falta de treinamento do músculo do soalho pélvico } \\
\text { para o aumento de sua própria contratilidade. }\end{array}$ & $\begin{array}{l}\text { O treinamento do músculo do soalho pélvico é um } \\
\text { meio eficaz para o aumento de sua própria } \\
\text { contratilidade, tanto em mulheres grávidas } \\
\text { primigestas como em primíparas pós-parto. }\end{array}$ \\
\hline $\begin{array}{l}\text { Artigo 09:16 Incontinencia urinaria a los } 6 \\
\text { meses del parto. }\end{array}$ & $\begin{array}{l}\text { Estudo prospectivo } \\
\text { Nível de evidência: } \\
\text { IV }\end{array}$ & $\begin{array}{l}\text { 1) Manobra de Kristeller; } \\
\text { 2) Não recuperação do peso pré gestacional; } \\
\text { 3) Peso do bebê ao nascer > 3,5 kg; } \\
\text { 4) Parto vaginal instrumentalizado; } \\
\text { 5) Renda familiar. }\end{array}$ & $\begin{array}{l}\text { A IU é um problema de saúde pública. Faz-se } \\
\text { necessário a intervenção dos profissionais da saúde } \\
\text { para sua prevenção, bem como o incentivo da } \\
\text { prática de exercícios físicos e perineais, assim como } \\
\text { o controle do IMC. }\end{array}$ \\
\hline $\begin{array}{l}\text { Artigo } 10:{ }^{17} \text { Prevalência de incontinência } \\
\text { urinária e disfunção muscular do assoalho } \\
\text { pélvico em primíparas dois anos após } \\
\text { parto cesárea: estudo transversal. }\end{array}$ & $\begin{array}{l}\text { Estudo transversal } \\
\text { Nível de } \\
\text { Evidência: IV }\end{array}$ & $\begin{array}{l}\text { 1) Disfunção muscular do soalho pélvico por ganho } \\
\text { de peso durante a gestação; } \\
\text { 2) A IU durante a gestação aumenta o risco de IU nos } \\
\text { dois primeiros anos pós-parto; } \\
\text { 3) Peso do RN; } \\
\text { 4) Idade materna. }\end{array}$ & $\begin{array}{l}\text { O parto cesáreo eletivo não constitui uma ação de } \\
\text { prevenção para a IU. }\end{array}$ \\
\hline $\begin{array}{l}\text { Artigo } 11:{ }^{18} \text { Efecto de un programa de } \\
\text { ejercicio físico en la recuperación post- } \\
\text { parto. Estudio piloto. }\end{array}$ & $\begin{array}{l}\text { Estudo } \\
\text { experimental, tipo } \\
\text { randomizado, não } \\
\text { pareado, } \\
\text { controlado, não } \\
\text { cego. } \\
\text { Nível de evidência } \\
\text { II } \\
\end{array}$ & $\begin{array}{l}\text { 1) Não recuperação do peso pré gestacional; } \\
\text { 2) Não realização de exercícios físicos na gestação e } \\
\text { no pós parto; } \\
\text { 3) Desconfortos causados pelo parto. }\end{array}$ & $\begin{array}{l}\text { Exercícios perineais e atividade física durante a } \\
\text { gestação contribuem para se evitar a IU no } \\
\text { puerpério. }\end{array}$ \\
\hline
\end{tabular}

Rev Enferm Health Care [Online]. Jan/Jul 2020; 9(1):144-159

ISSN 2317-1154 


\begin{tabular}{|c|c|c|c|}
\hline $\begin{array}{l}\text { Artigo } 12:{ }^{19} \text { Impact of childbirth and } \\
\text { mode of delivery on vaginal resting } \\
\text { pressure and on pelvic floor muscle } \\
\text { strength and endurance. }\end{array}$ & $\begin{array}{l}\text { Estudo prospectivo } \\
\text { de coorte } \\
\text { Nível de evidência: } \\
\text { IV }\end{array}$ & $\begin{array}{l}\text { 1) Ausência ou pouco fortalecimento da musculatura } \\
\text { pélvica durante gestação; } \\
\text { 2) Parto vaginal com utilização de fórceps. }\end{array}$ & $\begin{array}{l}\text { A manutenção da força muscular pélvica é } \\
\text { importante para a continência urinária pós parto, } \\
\text { independente da via de parto. A incontinência } \\
\text { iniciada durante a gestação tende a diminuir no pós } \\
\text { parto. }\end{array}$ \\
\hline $\begin{array}{l}\text { Artigo } 13::^{20} \text { Double incontinence in a } \\
\text { cohort of nulliparous pregnant women. }\end{array}$ & $\begin{array}{l}\text { Estudo prospectivo } \\
\text { de coorte } \\
\text { Nível de evidência: } \\
\text { IV }\end{array}$ & $\begin{array}{l}\text { 1) IU na gestação; } \\
\text { 2) Parto vaginal; } \\
\text { 3) Utilização de instrumentais por ocasião do parto; } \\
\text { 4) Episiotomia; } \\
\text { 5) Histórico familiar. }\end{array}$ & $\begin{array}{l}\text { Durante as consultas de pré-natal, este assunto deve } \\
\text { ser explorado para que as mulheres se sintam a } \\
\text { vontade para expor a IU, e assim, receberem um } \\
\text { tratamento adequado durante o período gestacional e } \\
\text { puerpério, independente da via de parto. }\end{array}$ \\
\hline $\begin{array}{l}\text { Artigo } 14:{ }^{21} \text { The impact of gestational } \\
\text { diabetes mellitus on postpartum urinary } \\
\text { incontinence: a longitudinal cohort study } \\
\text { on singleton pregnancies. }\end{array}$ & $\begin{array}{l}\text { Estudo prospectivo } \\
\text { de coorte. } \\
\text { Nível de evidência: } \\
\text { IV }\end{array}$ & $\begin{array}{l}\text { 1) Diabete mellitus gestacional; } \\
\text { 2) Hipertensão Arterial gestacional associado ao uso } \\
\text { de anti-hipertensivo; } \\
\text { 3) Paridade; } \\
\text { 4) IMC; } \\
\text { 5) Idade. }\end{array}$ & $\begin{array}{l}\text { Diante deste achado, os profissionais de saúde } \\
\text { devem atentar para os cuidados de prevenção de IU } \\
\text { em gestantes com Hipertensão além de DMG, } \\
\text { adotando medidas além do controle glicêmico, } \\
\text { alimentação e orientações quanto aos riscos } \\
\text { causados por este agravo. }\end{array}$ \\
\hline $\begin{array}{l}\text { Artigo } 15: 22 \text { The onset, recurrence and } \\
\text { associated obstetric risk factors for } \\
\text { urinary incontinence in the first } 18 \\
\text { months after a first birth: an Australian } \\
\text { nulliparous cohort study. }\end{array}$ & $\begin{array}{l}\text { Estudo prospectivo } \\
\text { de coorte. } \\
\text { Nível de evidencia: } \\
\text { IV }\end{array}$ & $\begin{array}{l}\text { 1) Trabalho de parto prolongado; } \\
\text { 2) A IU durante a gravidez favorece a IU no pós } \\
\text { parto; } \\
\text { 3) Traumas causados pelo parto vaginal; } \\
\text { 4) Cesariana após o segundo estágio do trabalho de } \\
\text { parto. }\end{array}$ & $\begin{array}{l}\text { Deve ser avaliado o benefício de se manter a mulher } \\
\text { em trabalho de parto prolongado. } \\
\text { *O peso do bebê ao nascer não está } \\
\text { significativamente relacionado à IU. }\end{array}$ \\
\hline $\begin{array}{l}\text { Artigo } 16:{ }^{23} \text { Can the delivery method } \\
\text { influence lower urinary tract symptoms } \\
\text { triggered by the first pregnancy? }\end{array}$ & $\begin{array}{l}\text { Estudo prospectivo } \\
\text { longitudinal. } \\
\text { Nível de } \\
\text { Evidência: IV }\end{array}$ & $\begin{array}{l}\text { 1) A frequência, a urgência, a nictúria e a } \\
\text { incontinência de urgência, desencadeadas durante a } \\
\text { gestação, diminuíram significativamente no período } \\
\text { pós-parto, independentemente da via de parto; } \\
\text { 2) Os sintomas relacionados à perda urinária devido } \\
\text { ao esforço persistiram após o parto vaginal; } \\
\text { 3) Parto vaginal; } \\
\text { 4) IU durante a gestação. }\end{array}$ & $\begin{array}{l}\text { A incontinência iniciada durante a gestação tende a } \\
\text { diminuir no pós parto. Porém, no parto vaginal } \\
\text { tende a ser mais persistente. }\end{array}$ \\
\hline $\begin{array}{l}\text { Artigo } 17:{ }^{24} \text { Pelvic floor muscle exercises } \\
\text { utilizing trunk stabilization for treating } \\
\text { postpartum urinary incontinence: } \\
\text { randomized controlled pilot trial of } \\
\text { supervised versus unsupervised training. }\end{array}$ & $\begin{array}{l}\text { Estudo } \\
\text { randomizado, } \\
\text { duplo-cego estudo } \\
\text { controlado. }\end{array}$ & $\begin{array}{l}\text { 1) Falta de exercícios dos músculos do soalho } \\
\text { pélvico. }\end{array}$ & $\begin{array}{l}\text { 1) O exercício dos músculos do soalho pélvico } \\
\text { utilizando a estabilização do tronco sob supervisão } \\
\text { de fisioterapeuta pode ser benéfico para o manejo da } \\
\text { IU pós-parto. }\end{array}$ \\
\hline
\end{tabular}

Rev Enferm Health Care [Online]. Jan/Jul 2020; 9(1):144-159

ISSN 2317-1154 


\begin{tabular}{|c|c|c|c|}
\hline & $\begin{array}{l}\text { Nível de } \\
\text { Evidência: II }\end{array}$ & & \\
\hline $\begin{array}{l}\text { Artigo } 18:{ }^{25} \text { Prevalência de incontinência } \\
\text { urinária autorreferida no pós-parto e } \\
\text { fatores relacionados. }\end{array}$ & $\begin{array}{l}\text { Estudo } \\
\text { exploratório, com } \\
\text { coleta transversal } \\
\text { dos dados. } \\
\text { Nível de } \\
\text { Evidência: IV }\end{array}$ & $\begin{array}{l}\text { 1) Cor branca; } \\
\text { 2) Sobrepeso; } \\
\text { 3) Parto vaginal; } \\
\text { 4) Episiotomia; } \\
\text { 5) Primiparidade; } \\
\text { 6) Não houve diferença entre as mulheres que } \\
\text { receberam e que não receberam orientações de } \\
\text { preparo do períneo durante a gestação e pós parto. }\end{array}$ & $\begin{array}{l}\text { A IU ocorre entre o primeiro e o terceiro mês sendo } \\
\text { mais comum a IUE e do tipo moderada, interferindo } \\
\text { no cotidiano. Contudo, investigação e a promoção } \\
\text { de ações para prevenção e ou tratamento amenizam } \\
\text { os desconfortos causados pela IU. }\end{array}$ \\
\hline
\end{tabular}

Fonte: Elaborado pelas autoras 


\section{DISCUSSÃO}

O surgimento de IU pode ser decorrente a vários fatores, desde situações pré-gestacionais, alterações fisiológicas da gestação, o parto e o puerpério.

Em relação às condições preexistentes, estas são citadas nos Artigos 4, 5, 6, 7, 9, 10, 14 e 18. O Artigo 5 cita doenças como asma, hipertensão arterial sistêmica e constipação intestinal. Artigo sobre incontinência urinária ${ }^{5}$ não cita asma, mas se refere à bronquite crônica como fator associado ao quadro. Uma explicação para tal achado é a tosse, sintoma presente tanto na asma quanto na bronquite e, considerando-se que a tosse está diretamente relacionada à incontinência de esforço, ao tossir ocorre o aumento da pressão na bexiga. Como o ato de fumar pode ser um fator que desencadeie a tosse, este é considerado como condição que contribui para o desenvolvimento de $\mathrm{IU}^{26}$ Por analogia, a constipação intestinal pode ser também a explicação para a incontinência de esforço, mencionada no Artigo 5. A presença de fezes no reto pode dificultar $o$ esvaziamento da bexiga, levando a instabilidade no músculo detrusor, podendo afetar o nervo. ${ }^{26}$ Dessa forma, medidas que evitem a constipação intestinal durante gravidez e puerpério devem ser encorajadas .

Os Artigos 4, 7, 10 e 14 destacam a idade materna como fator que está relacionado ao desenvolvimento de IU. Esta é uma questão mencionada na literatura ${ }^{27}$ corroborando a ideia de que a maioridade possa ser um risco de laceração decorrente de alterações fisiológicas na função nervosa, além do número de fibras musculares que diminuem com o passar do tempo.

Outro fator de risco da IU refere-se ao ganho de peso durante a gestação e a obesidade, abordado nos Artigos 6, 7, 18. Porém, o índice de massa corporal (IMC) aumentado (sobrepeso ou obesidade nos três diferentes graus) é um fator que pode ser modificado, como ponto de prevenção. ${ }^{27}$

O Artigo 13 aborda a questão de histórico familiar na IU durante a gravidez. Esse fato é corroborado em revisão de literatura sobre $\mathrm{IU}^{28}$, na qual os autores mencionam fatores hereditários na ocorrência desse agravo. Esse deve ser um ponto a ser pesquisado durante o pré-natal, no sentido de se buscar estratégias para a prevenção.

O Artigo 14 cita o Diabete Mellitus (DM) como fator relacionado à IU no pósparto. Artigo de revisão se refere à associação 
entre o DM e a IU ${ }^{28}$, uma vez que um possível aumento da vulnerabilidade do assoalho pélvico pode ocorrer devido a uma mudança do tecido biológico e da inervação do músculo pélvico, por ocasião do DM. Ainda o Artigo 14 destaca a hipertensão arterial gestacional e faz a associação ao uso do antihipertensivo Metildopa, devido seu mecanismo de ação, simpaticolítico de ação central, no qual atua diminuindo a pressão da uretra. Este achado é citado na literatura, sendo que os autores sugerem maiores investigações sobre este tema. ${ }^{29}$

No aspecto paridade, os Artigos 3, 5 e 14 citam que o número de partos interfere na musculatura pélvica, podendo desencadear a IU. A paridade por si só determina alterações na musculatura pélvica. ${ }^{30}$

As alterações fisiológicas ocorridas na gravidez conforme descrevem os Artigos 4, 6, $7,8,9,10,11,13,14,15$ e 16, podem desencadear modificações no assoalho pélvico. Durante a gravidez, os hormônios progesterona e relaxina, produzidos pelos ovários relaxam os ligamentos e músculos. $\mathrm{O}$ crescimento do feto determina o aumento da pressão intra-abdominal e os músculos do assoalho pélvico são estirados danificando a musculatura, outro fator que pode levar a incontinência urinária pós parto. ${ }^{31}$

Nesta revisão integrativa percebeu-se que não há consenso entre os autores sobre a relação do peso do recém-nascido $(\mathrm{RN})$ e o desenvolvimento de IU no puerpério. Os Artigos 9 e 10 citam o peso do RN como fator relacionado à IU no puerpério. Entretanto, o Artigo 15 discorda deste dado. Porém, o peso fetal, associado às alterações hormonais da gravidez sobre a musculatura do assoalho pélvico podem levar a IU, sendo o mecanismo exato ainda obscuro. ${ }^{32}$

O parto vaginal é citado pelos Artigos $4,7,15,16$ e 18 como agente relacionado à IU no período pós-parto. No entanto, este não deve ser considerado critério decisivo para a realização de cesárea. Artigo sobre $\mathrm{IU}^{33}$ aponta aspectos assistenciais que podem contribuir para a satisfação das mulheres e a necessidade de outras investigações para compreender melhor a multidimensionalidade do processo de parto, seja vaginal ou uma cesariana. Os autores fazem um apanhado de estudos que apresentam percepções positivas e negativas das mulheres sobre os dois tipos de parto. A literatura $^{34}$ postula que alguns agravos ocorridos no assoalho pélvico estão 
naturalmente relacionados ao trabalho de parto e que gravidez em si é um fator de risco para este músculo, podendo alterar a força muscular do assoalho pélvico. Além disso,

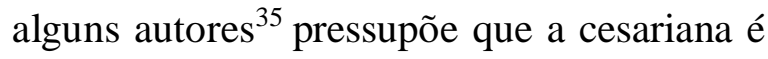
um procedimento que protege a mulher contra IU. Porém, a cesárea não é recomendada unicamente como preventivo para IU, pois há estudos ${ }^{26}$ apontando que a mesma não expressa uma clara redução de risco para incontinência. Ressalta-se que em nenhum dos artigos pesquisados houve o encorajamento de realização da cesárea unicamente como mecanismo de proteção do assoalho pélvico para evitar IU.

O Artigo 9 cita a Manobra de Kristeler como fator relacionado à IU no pós parto. Esta técnica caracteriza-se pela aplicação de uma pressão no fundo uterino durante o período expulsivo com objetivo de encurtálo. ${ }^{36}$ Porém, estratégias para diminuir a duração do segundo estágio do parto, tais como puxo dirigido e a manobra de Kristeler não devem ser realizadas. ${ }^{37}$

O parto instrumentalizado está relacionado a lesões na musculatura do assoalho pélvico como laceração de primeiro, segundo, terceiro e quarto graus, conforme os Artigos 4, 7, 11, 13 e 15. No entanto, há trabalho $^{26}$ que refere não haver a relação do parto instrumental com a ocorrência de IU.

Em relação a medidas de cuidados com o períneo durante o período expulsivo, o Ministério da Saúde $^{37}$ descata estudos que revelam que o uso de compressas mornas contribuem para um menor risco de lacerações e menor ocorrência de IU aos três meses após o parto. O uso de spray de Lidocaína e a massagem perineal durante o segundo estágio do parto resultam em menor incidência de lacerações de grau II e grau III respectivamente, mas não está recomendado para a redução da dor no segundo estágio do parto. $^{37}$

Quanto a situações ocorridas no puerpério, os Artigos 1, 6, 9, 12, 17 e 18 discorrem sobre a influência dos exercícios musculares destinados ao assoalho pélvico na prevenção e tratamento da incontinência durante e após a gravidez. Há referencias ${ }^{38}$ de pontos positivos da atividade física durante a gravidez, na prevenção da IU, como os exercícios de intensidade leve à moderada. Esses exercícios apresentam melhor resultado se forem realizados sob supervisão de um profissional especializado. ${ }^{39-40}$ Em contrapartida, há situações que contra indicam a prática de atividade física na 
gestação, sendo estas: hipertensão arterial, anemia, doenças tireoidianas, descontrole metabólico, obesidade mórbida e história de sedentarismo extremo. ${ }^{39}$ Nessas condições, a gestante deve ser muito bem orientada por profissionais do ramo de atividades físicas. Lembra-se que a gravidez e o parto são condições fisiológicas naturais, mas que causam alterações emocionais e físicas nas mulheres. ${ }^{41}$

Os achados desta revisão indicam que a gestação por si só constitui fator de risco para IU no puerpério. Recomenda-se que os profissionais de saúde que prestam cuidados a mulher incorporem um histórico de perda urinaria durante o acompanhamento prénatal. Os artigos incluídos nesta revisão sugerem que esforços para prevenir a IU no puerpério devem começar durante a gravidez.

\section{CONCLUSÃO}

De acordo com o estudo realizado, foi possível encontrar um consenso ente os fatores que mais favorecem a IU pós parto. Dos três fatores mais prevalentes, em primeiro lugar, estão as alterações fisiológicas da gravidez. Em seguida, aparecem condições preexistentes, que na maioria das vezes são inevitáveis, como as doenças e o histórico familiar. Outro fator importante foi à falta de orientações e o não preparo da musculatura pélvica, sendo que este preparo consiste em medidas simples e fáceis de serem aplicadas. $\mathrm{O}$ agravante é que nem sempre as gestantes são abordadas nestes aspectos. (por desconhecimento da relevância e magnitude do problema por parte dos profissionais)

A colaboração dos profissionais de saúde com medidas de orientações prevenção para o aparecimento ou mesmo para minimizar o agravo, pode devolver a paciente a auto estima além da esperança de que poderá voltar as suas atividades de vida diária com a segurança e auto controle físico evitando um desconforto e uma preocupação a mais nesse período.

O enfermeiro estomaterapeuta, atuando de forma ativa durante a gestação com atitudes preventivas, contribui positivamente em todas as situações do contexto: estado pré-gravídico, gravídico, parto e puerpério contribuindo com o autoconhecimento da mulher e a satisfação de um auto controle oferecido durante as consultas e avaliações necessárias.

\section{REFERÊNCIAS}


1. Andrade RD, Santos JS, Maia MAC, Mello DF. Fatores relacionados à saúde da mulher no puerpério e repercussões na saúde da criança. Esc Anna Nery. 2015;19(1):1816.

2. Leroy LS, Lopes MHBM. A incontinência urinária no puerpério e o impacto na qualidade de vida relacionada à saúde. Rev. Latino-Am. Enfermagem. 2012;20(2):[08 telas]

3. Rohr G, Christensen K, Ulstrup K, Kragstrup J. Reproducibility and validity of simple questions to identify urinary incontinence in elderly women. Acta Obstet Gynecol Scand. 2004;83(10):969-72.

4. Glazener CM, Herbison GP, MacArthur C, Lancashire R, McGee MA, Grant AM, Wilson PH. New postnatal urinary incontinence: obstetric and other risk factors in primiparae. BJOG. 2006;113(2):208-17. 5. Sacomori C, Bôer L, Sperandio FF, Cardoso FL. Prevalência e variáveis associadas à incontinência urinária no terceiro trimestre gestacional. Rev. Bras. Saude Mater. Infant. 2013;13(3):215-21. 6. Mendes KDS, Silveira RCCP, Galvão CM. Revisão integrativa: método de pesquisa para a incorporação de evidências na saúde e na enfermagem. Texto contexto enferm. 2008;17(4): 758-64.

7. Galvão CM, Sawada NO. Prática baseada em evidências: estratégias para sua implementação na enfermagem. Rev. bras. enferm. 2003;56(1):57-60.

8. Moossdorff-Steinhauser HF, AlbersHeitner P, Weemhoff M, Spaanderman ME, Nieman FH, Berghmans B. Factors influencing postpartum women's willingness to participate in a preventive pelvic floor muscle training program: a web-based survey. Eur J Obstet Gynecol Reprod Biol. 2015; 195:182-7.

9. Brown S, Gartland D, Perlen S, MacArthur C. Consultation about urinary and faecal incontinence in the year after childbirth: a cohort study. BJOG. 2015; 122(7):954-62.

10. Viñaspre Hernández RR, Aznar CT, Aranda ER. Factors associated with treatment-seeking behavior for postpartum urinary incontinence. J Nurs Scholarsh. 2014; 46(6):391-7.

11. Martin-Martin S, Pascual-Fernandez A, Alvarez-Colomo C, Calvo-Gonzalez R, Muñoz-Moreno M, Cortiñas-Gonzalez JR. Urinary incontinence during pregnancy and postpartum. Associated risk factors and influence of pelvic floor exercises. Arch Esp Urol. 2014; 67(4):323-30.

12. Pavón AG, Chao CW, Rodriguez NR, Iglesias FJV. Prevalencia y factores de riesgo de incontinencia urinaria en mujeres que consultan por dolor lumbopélvico: estudio multicéntrico. Atención Primaria. 2014; 46(2):100-8.

13. Viñaspre Hernández RR, Aranda ER, Aznar CT Urinary incontinence and weight changes during pregnancy and post partum: a pending challenge. Midwifery. 2013; 29(12):123-9.

14. Chan SS, Cheung RY, Yiu KW, Lee LL, Chung TK. Prevalence of urinary and fecal incontinence in Chinese women during and after their first pregnancy. Int Urogynecol J. 2013; 24(9):1473-9.

15. Marques J, Botelho S, Pereira LC, Lanza $\mathrm{AH}$, Amorim CF, Palma P, Riccetto C.

Pelvic floor muscle training program increases muscular contractility during first pregnancy and postpartum: electromyographic study. Neurourol Urodyn. 2013; 32(7):998-1003. 
16. Hernández RRV, Aranda ER, Aznar CT. Incontinencia urinaria a los 6 meses del parto. Medicina Clínica. 2013; 141(4): 14551.

17. Barbosa AMP, Marini G, Piculo F, Rudge CVC, Calderon IMP, Rudge MVC. Prevalência de incontinência urinária e disfunção muscular do assoalho pélvico em primíparas dois anos após parto cesárea: estudo transversal. Sao Paulo Med J. 2013; 131(2):95-9.

18. Barakat R, Bueno C, Durana AD, Coterón J, Montejo R. Efecto de un programa de ejercicio físico en la recuperación post-parto. Estudio piloto. Arch Med Deporte. 2013; 30(2):96-101. 19. Hilde G, Stær-Jensen J, Siafarikas F, Engh ME, Brækken IH, Bo K.Impact of childbirth and mode of delivery on vaginal resting pressure and on pelvic floor muscle strength and endurance. Am J Obstet Gynecol. 2013; 208(1):50-7.

20. Espuña-Pons M, Solans-Domènech $M$, Sánchez E. Double incontinence in a cohort of nulliparous pregnant women. . Neurourol Urodyn. 2012; 31(8):1236-41.

21. Chuang CM, Lin IF, Horng HC, Hsiao YH, Shyu IL, Chou P. The Impact of Gestational Diabetes Mellitus on Postpartum Urinary Incontinence: A Longitudinal Cohort Study on Singleton Pregnancies. BJOG. 2012; 119(11):1334-1343.

22. Gartland D, Donath S, MacArthur, Brown FJ. The onset, recurrence and associated obstetric risk factors for urinary incontinence in the first 18 months after a first birth: an Australian nulliparous cohort study. BJAG. 2012; 119(11):1361-9.

23. Botelho S, Silva JM, Palma P, ; Herrmann V, Riccetto C. Can the delivery method influence lower urinary tract symptoms triggered by the first pregnancy? Int. braz j urol. 2012; 8(2):267-76.
24. Kim EY, Kim SY, Oh DW. Pelvic floor muscle exercises utilizing trunk stabilization for treating postpartum urinary incontinence: randomized controlled pilot trial of supervised versus unsupervised training. Clin Rehabil. 2012; 26(2):132-41. 25. Lopes DBM, Praça NS. Prevalência de incontinência urinária autorreferida no pósparto e fatores relacionados. Acta paul. enferm. 2012; $25(4)$ ): 574-580.

26. Wesnes SL, Lose G. Preventing urinary incontinence during pregnancy and postpartum: a review. Int Urogynecol J. 2013; 24(6):889-99.

27. Sangsawang B, Sangsawang N. Stress urinary incontinence in pregnant women: a review of prevalence, pathophysiology, and treatment. Int Urogynecol J. 2013; 24(6):901-12.

28. Higa R, Lopes MHBM, Reis MJ. Fatores de risco para incontinência urinária na mulher. Rev Esc Enferm USP. 2008; 42(1):187-92.

29. Chuang CM, Lin IF, Hsiao YH, Shuu IL, Chou P. The impact of gestational diabetes mellitus on postpartum urinary incontinence: a longitudinal cohort study on singleton pregnancies. BJOG. 2012; 119(11):1334-43. 30. Pavón AG, Chão CW, Rodriguez NR, Iglesias FJG. Prevalencia y factores de riesgo de incontinencia urinaria en mujeres que consultan por dolor lumbopélvico: estudio multicêntrico. Atención Primaria. 2014; 46(2):100-8.

31. Steen M. Promoting continence in women following childbirth. Nurs Stand. 2013; 28(1):49-57.

32. Sangsawang B. Risk factors for the development of stress urinary incontinence during pregnancy in primigravidae: a review of the literature. Eur J Obstet Gynecol Reprod Biol. 2014; 178:27-34. 
33. Velho MB, Santos EKA, Brüggemann OM, Brígido VC. Vivência do parto normal ou cesáreo: revisão integrativa sobre a percepção de mulheres. Texto Contexto Enferm. 2012; 21(2): 458-66.

34. Low LK, Miller JM, Guo Y, AshtonMiller JA, DeLancey JO, Sampselle CM. Spontaneous pushing to prevent postpartum urinary incontinence: a randomized, controlled trial. Int Urogynecol J. 2013; 24(3):453-60. 35. Azam S, Khanam A, Tirlapur S, Khan K. Planned caesarean section or trial of vaginal delivery: a meta-analysis. Curr Opin Obstet Gynecol. 2014; 26(6):461-8.

36. Organização Mundial da Saúde. Assistência ao parto normal: um guia prático. Genebra: OMS, 1996.

37. BRASIL. Ministério da Saúde. Secretaria de Ciência, Tecnologia e Insumos Estratégicos. Comissão Nacional de Incorporação de Tecnologias no SUS. Diretriz nacional de assistência ao parto normal: relatório de recomendação. Brasília, DF; $2016 b$.

38. Lima FR, Oliveira N. Pregnancy and exercise. Rev. Bras. Reumatol. 2005; 45(3):188-90.
39. Sánchez-García JC, Rodríguez-Blanque R, Villar NM, Sánchez-López AM, Hernández MCL, Aguilar-Cordero MJ. Influencia del ejercicio físico sobre la calidad de vida durante el embarazo y el pós-parto: revisión sistemática. Nutr Hosp. 2016; 33(Supl. 5):1-9. Disponible in: http://revista.nutricionhospitalaria.net/index. $\mathrm{php} / \mathrm{nh} /$ article/view/514

40. Assis LC. Evolução da função muscular do assoalho pélvico no pós-parto [tese]. Botucatu (SP): Faculdade de Medicina de Botucatu; 2013.

41. Henz GS, Medeiros CRG, Salvatori M. A inclusão paterna durante o pré-natal. Rev Enferm Atenção Saúde. 2017; 6(1):52-66.

RECEBIDO: 25/10/2017

APROVADO: 06/12/2018

PUBLICADO: 07/2020 